\title{
Representaciones del proceso de diseño: de la didáctica a la metáfora
}

Úrsula Bravo ${ }^{(1)}$ y Erik Bohemia ${ }^{(2)}$

Resumen: Las representaciones visuales del proceso de diseño son usadas como dispositivos didácticos para ilustrar cuáles son las principales etapas, los tipos de pensamiento involucrados y los ciclos de retroalimentación que tienen lugar a lo largo del proceso. Considerando que la acción de representar implica seleccionar o enfatizar algunos elementos del fenómeno representado por sobre otros, nos preguntamos qué concepciones del diseño comunican esos modelos y qué aspectos omiten. Proponemos un estudio teórico con el objetivo de discutir dos aspectos centrales: a) la función didáctica de estos modelos y b) las diferentes narrativas o metáforas que estos expresan. Hemos seleccionado a discreción diferentes representaciones del proceso de diseño desde fuentes académicas y profesionales, incluyendo artículos y libros en formato físico y digital. Concluimos que estos modelos contribuyen a producir y reproducir una cultura profesional del diseño, en la medida que su creación, difusión y consumo permiten transmitir "valores compartidos" y una "narrativa" acerca del diseño entre diseñadores profesionales y en formación, pero también entre profesionales de otras áreas que buscan adoptar la forma de trabajar, pensar o aprender de los diseñadores.

Palabras clave: pensamiento de diseño - proceso de diseño - representación visual - imagen didáctica.

[Resúmenes en inglés y portugués en la página ]

(1) Úrsula Bravo. Diseñadora de la Pontificia Universidad Católica de Chile, Magister en Humanidades de la Universidad del Desarrollo, actualmente se encuentra estudiando el Doctorado en Educación de la Pontificia Universidad Católica de Chile. Su área de interés se relaciona con el Design Literacy. Actualmente está investigando la aplicación del pensamiento de diseño en el contexto escolar y la visualidad en el espacio escolar desde la perspectiva de las estéticas cotidianas. Universidad del Desarrollo, Chile / ubravo@udd.cl. Pontificia Universidad Católica de Chile / ursula.bravo@uc.cl.

(2) Erik Bohemia. Diseñador Industrial de RMIT University (Royal Melbourne Institute of Technology), Australia y Ph.D de la Universidad New South Wales, Australia. Sus áreas de interés se relacionan con el diseño como práctica cultural y los efectos materiales del diseño. Actualmente está investigando cómo los elementos culturales configuran los enfoques de los diseñadores y sus maneras de resolver los "problemas", así como el impacto cultural de las “soluciones" que desarrollan. Oslo Metropolitan University - erik.bohemia@oslomet.no 


\section{Introducción}

En los últimos años, el denominado design thinking ha trascendido las fronteras de la disciplina del diseño, siendo difundido entre profesionales de otras áreas como un método de resolución creativa de problemas. El propósito ha sido fomentar la identificación de problemas con foco en las necesidades y motivaciones de las personas, y el desarrollo de soluciones innovadoras que generen valor en términos económicos o sociales (Dorst, 2011; Hassi y Laakso, 2011; Johansson-Sköldberg, Woodilla, y Cetinkaya, 2013). En este contexto, las representaciones gráficas del proceso de diseño han sido herramientas visuales recurrentes porque explican de manera didáctica sus principales etapas, los tipos de pensamiento involucrados y los ciclos de retroalimentación que tienen lugar a lo largo del proceso.

Estos diagramas varían tanto en la estructura formal como en el número, nombre y nivel de especificidad de las diferentes etapas. Las que pueden ir desde tareas muy precisas hasta fases de gran complejidad que reúnen varias actividades y que se superponen entre sí. Los nombres con que se identifican las diferentes etapas pueden referirse tanto a procedimientos prácticos, como a habilidades cognitivas o socioemocionales (Bravo, 2016). La adaptación de estos modelos a otros campos profesionales ha generado nuevas representaciones del proceso de diseño, como los modelos propuestos por las organizaciones Design for Change, Index Design to Improve Life, Henry Ford Learning Institute y el manual Design Thinking for Educators desarrollado por IDEO, especialmente desarrollados para el ámbito de la educación escolar.

Considerando que la acción de representar implica seleccionar o enfatizar algunos elementos del fenómeno representado por sobre otros, nos preguntamos qué concepciones del diseño comunican esos modelos y qué aspectos omiten. La relevancia de esta pregunta se relaciona con el uso didáctico que se da a estos modelos, ya que no solo tienen la función de representar cómo es el proceso de diseño, sino también guiar su ejecución. En este sentido, afirmamos que las representaciones visuales del pensamiento de diseño contribuyen a configurar y difundir una determinada cultura del diseño: es decir, comunican conceptos e ideas acerca de lo que significa el diseño, diseñar y ser diseñador. Proponemos un estudio teórico con el objetivo de discutir dos aspectos de las representaciones visuales del proceso de diseño: a) su función como recursos didácticos, y b) las diferentes narrativas o metáforas que estos modelos expresan. Comenzaremos analizando la función didáctica, sus sesgos y limitaciones, para luego proponer algunas metáforas que subyacen a estas representaciones. Ilustraremos nuestro análisis con diferentes modelos recogidos desde la literatura académica y profesional.

\section{La mediación didáctica de la visualización esquemática}

La realidad se presenta como un continuo compuesto por una multiplicidad de fenómenos de naturaleza diversa, muchos de los cuales ni siquiera son accesibles a los sentidos, dificultando su comprensión. En este contexto, la visualización a través de esquemas o diagramas opera como una mediación didáctica que busca hacer comprensibles fenómenos 
complejos a través de recursos gráficos (Costa, 1998) teniendo como propósito facilitar el procesamiento de la información y contribuir a mejorar su codificación y organización (Díaz-Barriga y Hernández, 2002). En definitiva, se trata de reducir la complejidad cognitiva, la ambigüedad y la incertidumbre de los fenómenos de la realidad, contribuyendo a su comprensión a través de un lenguaje visual esquemático. Así, por ejemplo, un diagrama de flujo entrega una descripción visual de la secuencia de actividades que tienen lugar a lo largo de un determinado proceso, contribuyendo a explicar en qué consiste dicho proceso, pero también guiando su ejecución.

Según Costa (1998), los esquemas constituyen un lenguaje que se caracteriza por ser lógico, estructurado, codificado, abstracto y de naturaleza unívoca o monosémica. Esta aparente racionalidad podría hacernos pensar que se trata de un lenguaje objetivo y neutro, sin embargo, como toda representación visual, los esquemas distan de ser meros reflejos de la realidad y no tienen un significado intrínseco: sus creadores les impregnan un significado que solo puede ser traducido por aquellos que comparten los mismos códigos culturales (Hall, 1997). Dentro de dichos códigos culturales, están incluidos los códigos visuales. Así, solo quienes entienden las convenciones de la representación, están habilitados para leerlas (Banks, 2007). Por ejemplo, la representación visual del tiempo ha incorporado convenciones espaciales y visuales. El tiempo pasado se relaciona con lo que quedó "atrás" y el tiempo futuro con lo que viene hacia "adelante". Se asume, entonces, que si una persona está de pie, le da la espalda al pasado y su rostro enfrenta el futuro (Meirelles, 2013). En términos gráficos, esto se traduce en líneas horizontales que se "leen" de izquierda a derecha, donde el extremo izquierdo representa el inicio y el derecho representa el fin: por tanto, un evento que ocurre antes debe ubicarse a la izquierda de otro que sucede después ${ }^{1}$. Chaplin (en Banks, 2007:17) caracteriza la función representacional relevando los siguientes aspectos: a) la forma de la representación no está dictada por la cosa representada sino por un conjunto de códigos o convenciones que solo son comprensibles para los observadores inmersos en una misma convención, b) la representación refleja y constituye un proceso social y c) la representación tiene agencia, entendida como algún tipo de fuerza intencional. Estos tres principios los podemos ejemplificar de la siguiente manera: a) las representaciones del proceso de diseño adoptan las convenciones visuales para la representación del tiempo, b) son utilizadas para socializar determinadas interpretaciones de lo que es el diseño y c) generan acciones que intervienen la realidad porque guían la ejecución del proceso de diseño.

Así, la representación visual contiene y transmite valores compartidos, códigos culturales, conceptos y emociones asociados al fenómeno representado; forma parte de las prácticas que produce y reproduce la cultura (Hall, 1997:xvii), incluidas las "pequeñas culturas" o culturas profesionales (Holliday, 1999). La representación del tiempo es un buen ejemplo de cómo codifican visualmente las diferentes culturas profesionales: aunque el tiempo histórico en occidente se lee de izquierda a derecha, en un diagrama de flujo el tiempo suele transcurrir de arriba hacia abajo, mientras que el tiempo geológico se lee de abajo hacia arriba en concordancia con las capas de sedimento que se hallan en una excavación. Hall (1997) sugiere que damos sentido a las cosas mediante las representaciones que hacemos de ellas: las palabras que usamos para describirlas, las historias que contamos sobre ellas, las imágenes que producimos. De esta manera, la representación opera clasificando, 
conceptualizando y asignando valores a la cosa representada. En este sentido, el diseño es la "cosa" a la que se le da un significado mediante diferentes representaciones visuales del proceso de diseñar. Estas representaciones, entonces, no solo ilustran un proceso, sino que además transmiten conceptos e ideas acerca del diseño, operando como recursos que permiten iniciar a los "no iniciados" (Bobbe, Krzywinski, y Woelfel, 2016; Howard, Culley, y Dekoninck, 2008), señalándoles qué es el diseño y cómo diseñar. Así se va configurando y sustentando una "cultura del diseño". Por lo tanto, no es lo mismo representar -ni entender- el diseño como un diagrama de flujo o como un ciclo de mejora continua. Cada forma de representar enfatiza diferentes aspectos del diseño.

Tal vez debido a este componente interpretativo y a la diversidad de vínculos disciplinares que tiene el diseño, es que existen tantas maneras diferentes de representar el proceso de diseño. En efecto, en la literatura académica y profesional del diseño, la ingeniería, los negocios, la innovación y la educación abundan las representaciones visuales del proceso de diseño. Son tantas y tan diversas, que hasta parecieran representar fenómenos diferentes, pudiendo generar confusión entre las personas que acuden a ellas buscando guiar un proceso creativo. Cross (2005) distingue entre modelos descriptivos y prescriptivos: los primeros describen las etapas o actividades más frecuentes realizadas por un diseñador, mientras que los otros definen un patrón óptimo para motivar a los diseñadores a trabajar de manera más eficiente y sistemática.

Las representaciones varían tanto en su estructura formal como en el número, nombre y nivel de especificidad de las diferentes etapas. Hay representaciones lineales, circulares, con forma de espiral o de matriz; algunas identifican un número determinado de etapas, en cambio otras visualizan el proceso como un continuo que atraviesa por dos grandes ciclos de divergencia y convergencia. Los nombres de las etapas pueden referirse a procedimientos prácticos -como prototipar, testear, comunicar-, a habilidades cognitivas-como analizar, sintetizar y definir-, y a actitudes o habilidades socioemocionales requeridas como la empatía- (Bravo, 2016). Esta diversidad ha sido observada por diversos autores (Bobbe et al., 2016; Dubberly, 2005; Gericke \& Blessing, 2012; Wynn \& Clarkson, 2005) y queda en evidencia a través del estudio de Wölfel, Debitz, Krzywinski, y Stelzer (2012), quienes recolectaron 701 modelos diferentes entrevistando a 50 diseñadores (es decir, cada diseñador se refirió en promedio a 14 modelos diferentes).

Dubberly (2005) clasificó más de 100 modelos según sus características formales o por el contexto a partir del cual se elaboraron: academia, consultoría profesional, desarrollo de software, etc. Gericke y Blessing (2012) comparan 23 modelos provenientes de diferentes disciplinas, incluidos la ingeniería mecánica, el diseño industrial y de softwares, la ingeniería de sistemas y de servicios, y la arquitectura, entre otros. Bobbe, Krzywinski y Woelfel (2016) comparan 15 modelos de procesos de diseño de instituciones académicas, organizaciones profesionales y estudios de diseño identificando cinco etapas principales. Beausoleil (2016) estudia diferentes modelos de procesos de innovación y propone una lista de competencias clave de innovación en tres niveles diferentes: conocimiento, aptitudes y habilidades. Bravo (2016) compara diez modelos de procesos de diseño provenientes del medio profesional con seis adaptados a la educación escolar, identificando cuatro etapas principales en modelos profesionales y seis en modelos de uso escolar. Howard y colaboradores (Howard et al., 2008) proponen una integración entre el proceso de diseño 
de ingeniería y el proceso creativo establecido a partir de la psicología cognitiva, afirmando que, aunque los procesos de diseño observados en la práctica son más erráticos de lo que la mayoría de las representaciones sugieren, ayudan a los ingenieros a utilizar mejor las herramientas creativas, metodológicas y técnicas.

En la medida que son usados para enseñar a estudiantes de diseño (Dubberly, 2005) -pero también escolares y a profesionales de otras áreas-, estas representaciones actúan como dispositivos didácticos ${ }^{2}$. Sin embargo, tienen limitaciones relacionadas con la selección, la interpretación y el énfasis dado a los elementos que conforman el fenómeno del diseño (a esto podríamos denominar sesgo de representación), pero también a los códigos visuales que dan forma a la representación (que llamaremos sesgo gráfico). Por ejemplo, un frío diagrama de flujo geométrico, lineal y monocromático no comunica lo mismo acerca del diseño que un diagrama compuesto por círculos de colores, en el que las líneas que representan los ciclos de iteración parecen cintas de colores que vuelan al viento, evocando festividad y optimismo. Del mismo modo, una estructura recta, no comunica lo mismo que una circular o una en forma de matriz (Figura 1). La estructura lineal sugiere causalidad, enfatiza el resultado, mientras que una estructura circular o espiral comunica ciclo, mejoramiento, evolución. La matriz sugiere lógica, categorías excluyentes.

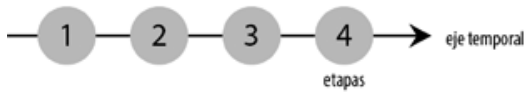

a) Estructura lineal recta

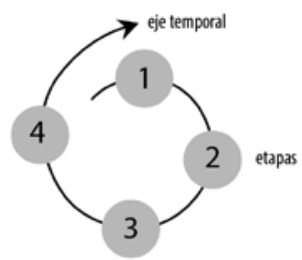

b) Estructura lineal circular

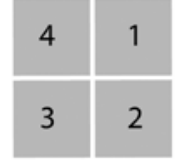

c) Estructura de matriz

Figura 1. El proceso de diseño representado con diferentes estructuras geométricas.

\section{Visualizar la complejidad del proceso de diseño}

¿Es posible atrapar en un diagrama la complejidad del proceso de diseño?

Suele decirse que un proceso de diseño comienza con la detección de un problema, una necesidad o una oportunidad (Ulrich y Eppinger, 2000) y termina en una solución (Figura 2a). Esta concepción positivista del diseño ha sido criticada por su carácter lineal, reduccionista y causal (Teal, 2010). Curiosamente, pese a que la complejidad que caracteriza al proceso de diseño ha sido ampliamente abordada en la literatura, rara vez se refleja en las representaciones visuales del proceso de diseño, que suelen comunicar una idea bastante más simple de lo que es el diseño y de lo que supone diseñar. Howard y colaboradores (2008) afirman que los procesos de diseño son más erráticos de lo que la mayoría de las 
representaciones sugieren, mientras que Dubberly (2005) sostiene que el solo hecho de registrar un proceso de diseño, no representa necesariamente lo que ocurrió ni por qué ocurrió. El autor establece una analogía entre las representaciones visuales del proceso de diseño y la selección que realiza un fotógrafo al tomar una fotografía: en efecto, el autor - de la fotografía o del proceso de diseño- es quien elige dónde apuntar la cámara: dónde comenzar y dónde terminar de trazar el proceso, con cuánto detalle describirlo, qué incluir y qué omitir. Este proceso de edición o encuadre limpia un mundo desordenado, generando una ilusión de linealidad y causalidad (Dubberly, 2005).

La complejidad del proceso también se explica por las características de los problemas que abordan los diseñadores y de la intrincada relación entre el problema y la solución. En 1973 Rittel y Webber (1973)observaron que la comprensión del problema y la solución se afectan mutuamente: la información necesaria para comprender el problema depende de la idea de que una persona tiene para resolverlo. Del mismo modo, las características y limitaciones del posible efecto que genere una solución contribuye a redefinir el problema (Figura 2b). Desde esta perspectiva, la definición del problema es uno de los resultados del proceso de diseño. Cross (2013) observa que los diseñadores no interpretan el brief de diseño como especificaciones para desarrollar una solución, sino como un punto de partida para explorar y definir el problema. De hecho, Dorst y Cross (2001) sugirieron que los diseñadores no consideran el problema como un hecho objetivo; por el contrario, lo interpretan y construyen desde sus propios contextos, experiencias, capacidades y recursos, manipulándolo durante casi todo el proceso. En este sentido, el problema no es sustantivo, sino verbo: es decir, el diseñador no "encuentra" un problema dado, sino que "problematiza" la realidad partir de la información recolectada.

Además de la compleja transición entre el problema y la solución, es necesario puntualizar que en el diseño también se da una transición entre una situación "actual" y una situación hipotética "futura"; entre el "pensamiento analítico" orientado a entender el problema y la "síntesis creativa" orientada a generar una solución; entre el pensamiento "divergente" y el pensamiento "convergente"; entre el "saber" y el "hacer" entre una situación "concreta" de la vida real que requiere una solución y las ideas "abstractas" acerca de una posible la solución, y desde esa solución "imaginada" hacia su "materialización” mediante la producción e implementación (Dubberly, 2005).

La idea de transición entre dos etapas, dos momentos o dos tipos de pensamiento, inevitablemente sugiere linealidad, porque supone que una situación va a dar paso a la siguiente, lo cual puede ser fácilmente representado por una línea que une dos puntos en el espacio. Sin embargo Bonsiepe (1999) ha señalado que la idea del proceso de diseño como una secuencia lineal de decisiones está determinada por un racionalismo de tipo cartesiano que asume que el diseño se origina en una tabla rasa en la que se definen funciones que luego son traducidas a formas. No obstante, añade el autor, las funciones no son entidades físicas que residen en los productos, sino en el lenguaje: las formas no tienen una función inherente que pueda definirse objetivamente: "Sólo gracias a la intervención de un observador se determina la relación entre una forma y su función” (Bonsiepe, 1999:149). Es decir, el hecho de que la forma de la manilla de una puerta haga un guiño a la forma de la mano "señalando" que debe ser accionada por esta, no tiene que ver tanto con la forma de la manilla como con el hábito adquirido y compartido de abrir puertas. A nuestro entender, esta 
sería una visión fenomenológica del diseño, debido a que el diseñador está estrechamente involucrado en la interpretación del fenómeno (Figura 2c).

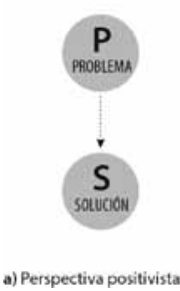

a) Perspectiva positivista

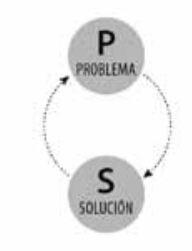

b) Perspectiva constructivista

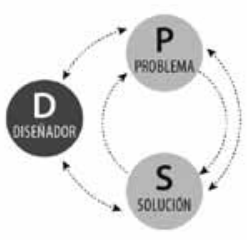

c) Perspectiva fenomenológica

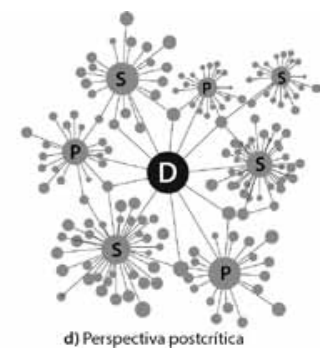

Figura 2: El proceso de diseño desde distintas perspectivas epistemológicas.

Teal (2010) coincide con Bonsiepe al criticar la concepción lineal del proceso de diseño. Según este autor, el diseñador (o el arquitecto) no se enfrenta a una simple relación entre medios y fines, sino que debe realizar una búsqueda que le permita delinear cuáles son los temas relevantes, las fuerzas y los efectos que se ponen en movimiento durante el proceso de diseño. Por esta razón, considera que la estructura rizomática (Figura 2d) -propuesta por Deleuze y Guattari- ofrece una mejor comprensión de los problemas de diseño, permitiendo una visualización de múltiples factores que interactúan en constante cambio y resaltando las interacciones -causales, secuenciales o compuestas- que ocurren constantemente durante el proceso de diseño. Cabe señalar, sin embargo, que la estructura rizomática puede servir para expresar la complejidad del diseño, pero difícilmente servirá para guiar un procedimiento.

\section{Metáforas subyacentes a las representaciones visuales del proceso de diseño}

Sintetizando el apartado anterior, podemos afirmar que la complejidad del proceso de diseño pareciera resistirse a ser representada por un solo esquema de carácter definitivo y universal. Las representaciones visuales del proceso de diseño operan enfatizando algunos aspectos por sobre otros y en este trabajo de selección y omisión transmiten una cierta narrativa de lo que el diseño es. Proponemos analizar dichas narrativas desde la perspec- 
tiva de las metáforas, entendidas como una comparación tácita que impregna al diseño de significados provenientes de otros fenómenos de la realidad.

Hace más de tres décadas, Morgan (1997 [1986]) exploró las organizaciones a través del lente de ocho metáforas. En el libro "Images of Organization", clasifica a las organizaciones como: a) máquinas, b) organismos vivos, c) cerebros, d) sistemas políticos, e) prisiones psíquicas, f) flujos, g) transformación, y h) instrumentos de dominación. En esta misma línea, Wilkes (Wilkes, 1989) sugiere que la metáfora es una herramienta especialmente apropiada para ayudarnos a apreciar, interpretar y comprender las complejidades, ambigüedades y paradojas asociadas con las organizaciones. Las metáforas contribuyen a una mejor comprensión de las organizaciones -incluidas sus posibilidades y limitaciones- y a la producción de culturas organizativas (Jermier y Forbes, 2011).

Nos gustaría analizar los modelos visuales del proceso de diseño desde la perspectiva de las metáforas, con el propósito de relevar (y revelar) cuáles son las narrativas o concepciones del diseño que subyacen a las representaciones visuales. Durante nuestra investigación, hemos identificado las siguientes metáforas que podrían ser útiles para explorar modelos de procesos de diseño:

- El diseño como un proceso racional

- El proceso de diseño como la trayectoria del pensamiento

- El diseño como proceso de evolución

- El proceso de diseño como ciclo de aprendizaje y mejora

No se trata de categorías excluyentes ni pretenden ser las únicas categorías posibles. Incluso los nombres que les hemos asignado pueden entenderse como provisorios. Pero lo proponemos como un punto de partida para futuros estudios que incluyan una aproximación empírica. En la siguiente sección discutiremos cada una de ellas.

\subsection{El diseño como un proceso racional}

Las representaciones del proceso de diseño desarrollados bajo la influencia de la ingeniería en contextos industriales, como la propuesta por French (en Cross, 2005:31) (Figura 3a) se centran fundamentalmente en la identificación de las principales etapas del proceso. Tienden a ser representaciones lineales, que se organizan en un eje temporal, tipo diagrama de flujo (de estructura vertical) o línea de tiempo (de estructura horizontal). En este eje se despliegan actividades o etapas. Algunos incluyen tareas, objetivos, resultados y ciclos de retroalimentación, que son diferenciados mediante recursos gráficos. Los modelos más sintéticos identifican solo las fases principales. La linealidad de estos esquemas sugiere causalidad y enfatiza la idea de un resultado final, en la medida que todas las etapas convergen en la etapa final, donde concluye el proceso.

Rittel y Webber (1973) observaron que los libros de ingeniería de sistemas comienzan con una enumeración de fases como 'comprender el problema o la misión', 'recopilar información', 'analizar información', 'sintetizar información y esperar el salto creativo, etc. Los autores advierten que frente a problemas complejos o "perversos", este tipo de esquema no fun- 
ciona, dado que no es posible entender el problema sin conocer su contexto. Por otro lado, advierten los autores, no es posible buscar información para entender el problema, sin considerar una posible solución. Es decir, no se puede entender primero, para resolver después. Lawson (2005) utilizó el proceso de diseño secuencial de RIBA (Figura 3b) para estudiar cómo podría ser interpretado por un diseñador. A nivel empírico, observó que resultaba difícil para un diseñador completar la fase 1 antes de comenzar la fase 2 . El autor sugiere que esta representación del proceso de diseño podría fomentar una dilatación improductiva ya que el modelo no indica cómo o cuándo realizar la transición entre una etapa y la siguiente, ni tampoco señala qué situaciones podrían gatillar el cambio.
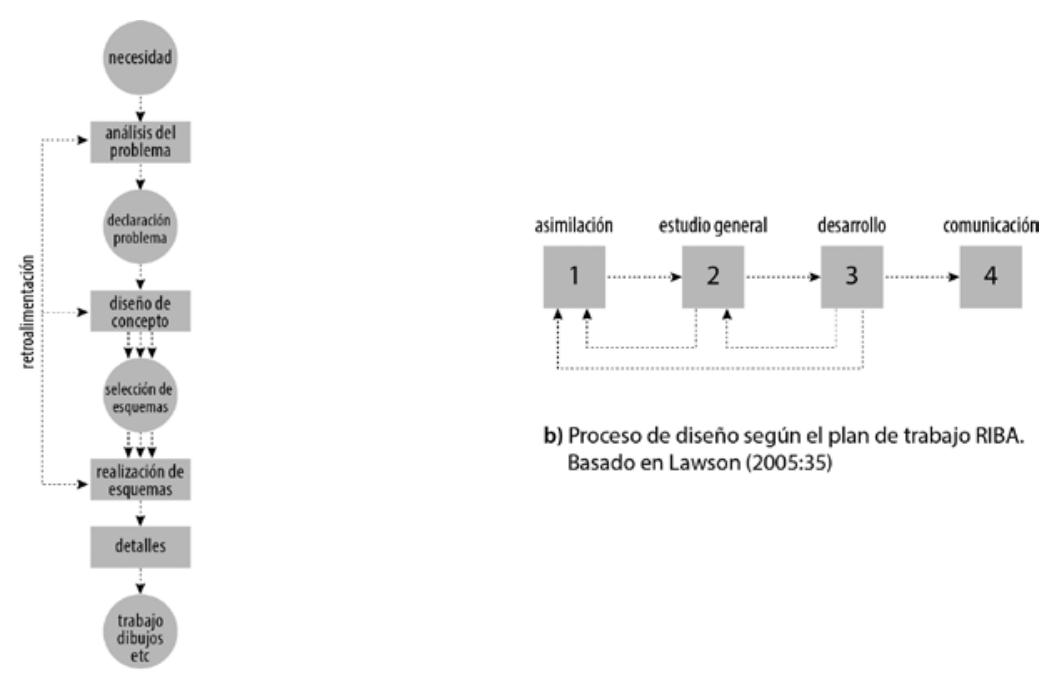

b) Proceso de diseño según el plan de trabajo RIBA. Basado en Lawson (2005:35)

a) Modelodel proceso de diseño de French (Cross, 2005:31)

Figura 3. Representaciones lineales que ilustran una secuencia de etapas que convergen en un resultado final.

\subsection{El proceso de diseño como la trayectoria del pensamiento}

Los diagramas de Alexander (1973), Dubberly, Evenson y Robinson (2008) y Kumar (2012), buscan visibilizar las formas de pensamiento involucradas en el diseño. Alexander (1973) distingue el contexto de la forma y explica que la complejidad del proceso se debe a las sucesivas interpretaciones mentales y formales que el diseñador hace del contexto para llegar a la forma (Figura 4a). Kumar (2012) establece dos ejes que se intersectan formando 
cuatro cuadrantes. El eje vertical que va de lo "real" a lo "abstracto" y el horizontal que va del "saber" al "hacer". Cada cuadrante está asociado con un tipo de pensamiento (análisis y síntesis) o de procedimiento (investigación y realización) (Figura 4b).

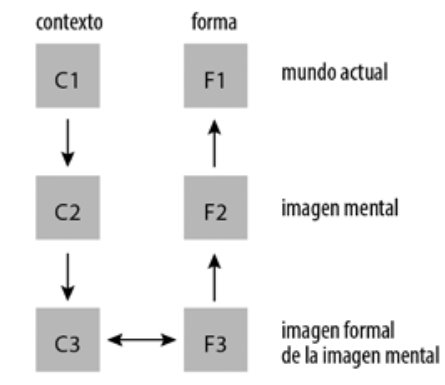

a) Basado en Christopher Alexander (1973:76)

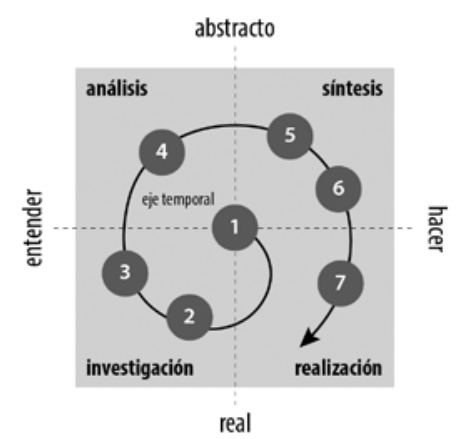

b) Basado en Design Innovation Process de Vijay Kumar (2013)

Figura 4. Los esquemas de Alexander (1973:76) y Kumar (2013) ilustran respectivamente la transición entre el contexto, la imagen mental y la forma (a) y la transición entre lo concreto o real, la abstracción del pensamiento analítico y sintético, para volver a lo real mediante la realización (b) pasando desde el entender hasta el hacer.

El modelo del doble diamante (Figura 5a) propuesto por el Design Council (Council Design et al., 2015) y el modelo "Dynamics of Divergence and Convergence" de Banathy (en Dubberly, 2005:24) (Figura 5b) combinan una estructura temporal lineal, con la representación de dos ciclos -los diamantes-compuestos por una primera etapa de pensamiento divergente y una segunda etapa de pensamiento convergente. En el primer ciclo del doble diamante el pensamiento divergente se asocia con la acción de descubrir y el convergente con definir el problema; mientras que, en el segundo ciclo, el pensamiento divergente se orienta a desarrollar la propuesta o solución, y el convergente con entregar (la propuesta). Por sus nombres en inglés, el modelo utiliza el recurso mnemónico de las $4 \mathrm{D}$ : discover, define, develop, delivery. Una diferencia importante entre ambos modelos, es que en el de Banathy (en Dubberly, 2005:24) atribuye la divergencia a la participación de personas provenientes de diferentes disciplinas. Sin embargo, el modelo no explicita de qué manera se logra la convergencia. 


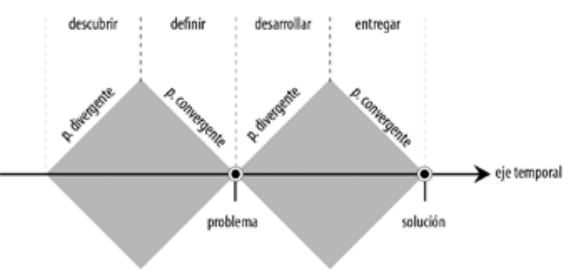

a) Basado en modelo de doble diamante de British Council (2015)

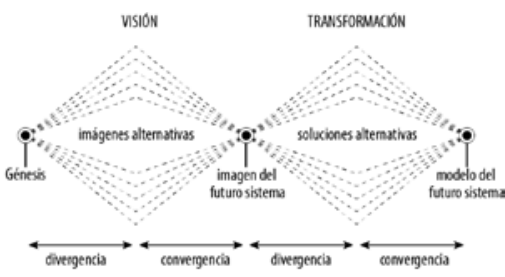

b) Basado en modelo de Bela H. Banathy (1996) según Dubberly (2004:24).

Figura 5. El modelo del doble diamante (a) y el modelo de Banathy (b) ilustran dos ciclos compuestos por una primera etapa de pensamiento divergente y una segunda etapa de pensamiento convergente.

En relación a este tipo de representación McComb, Kotovsky y Cagan (2014) han señalado que los diseñadores expertos rápidamente seleccionan una dirección determinada para desarrollar su propuesta, considerando menos posibilidades que los diseñadores novatos. En estos casos se lograrían niveles más bajos de divergencia y la convergencia se iniciaría mucho antes de lo que indica el modelo.

Además de la convergencia y la divergencia, muchos modelos representan de manera separada el análisis y la síntesis, asumiendo que se trata de operaciones cognitivas disociadas. No obstante, (Koberg \& Bagnall, 1981) afirman que tanto el análisis como la síntesis continúan a lo largo de todo el proyecto. Eastman (1970, citado por Lawson, 2004) y Akin (1986) respaldan este hecho señalando que el análisis (asociado a la comprensión del problema) está mucho más integrado con la síntesis (asociado a la generación de la solución) de lo que comúnmente se cree. Por ejemplo, Akin (1986) concluyó que los diseñadores generan constantemente nuevos objetivos y redefinen las restricciones, sugiriendo que el análisis está presente en todas las fases de diseño y la síntesis se encuentra en etapas tempranas del proceso. La implicación de entender estas actividades como actividades separadas tendría como inconveniente forzar a los miembros de un equipo de diseño a retrasar la creación de soluciones durante el mayor tiempo posible (Bason \& Austin, 2019).

\subsection{El diseño como proceso de evolución}

Maher, Poon, \& Boulanger (1996) afirman que el diseño no es un proceso de búsqueda, que comienza con un problema bien definido y termina en una solución (Figura 6a). Por el contrario, identifican el diseño con la exploración porque se inicia con un problema abierto y mal definido, y por tanto el diseñador, además de generar una solución, debe definir el problema (Figura 6b). Además, sugieren que es errado representar el proceso de diseño como una sucesión de etapas discretas o discontinuas y sostienen que el problema y la solución están interrelacionados y no son entidades separadas. Para caracterizar el 
proceso de diseño utilizan la analogía de la mutación genética, afirmando que el problema y la solución co-evolucionan o evolucionan de manera conjunta afectándose mutuamente (Figura 6c). Por ejemplo, la producción o testeo de un prototipo (espacio de solución) podría generar la necesidad de redefinir el problema (Dubberly, 2005), lo que a su vez generará un nuevo espacio de solución (Maher et al., 1996).

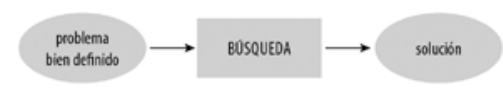

a) La búsqueda se inicia con un problema bien definido Basado en Maher, Poon yBoulanger (1996:4)

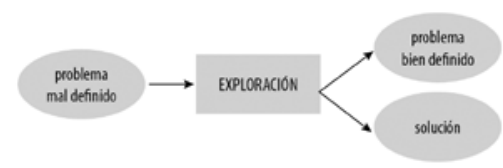

b) La exploración se inicia con un problema mal definido Basado en Maher, Poon y Boulanger (1996:4)

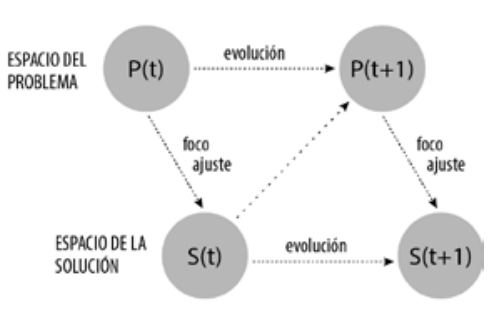

c) Modelo de coevolución del problema y la solución Basado en Maher, Poon y Boulanger (1996:7)

Figura 6: Maher, Poon y Boulanger discriminan entre búsqueda (a) y exploración (b), dependiendo del tipo de problema que de inicio al proceso (bien o mal definido). Además, proponen la metáfora de la coevolución entre el problema y la solución.

\subsection{El proceso de diseño como ciclo de aprendizaje y mejora}

El círculo y la espiral se han adoptado para ilustrar los ciclos de mejora continua. El movimiento Total Quality Metagaming (TQM) ha popularizado su modelo Shewhart-Deming Plan-Do-Study-Act Cycle utilizado para eliminar desperdicios en proceso de fabricación (1997). En el ámbito del diseño, los procesos cíclicos suponen evaluar la propuesta una vez que se ha implementado, para posteriormente introducir las mejoras necesarias. Una de las implicaciones de este tipo de modelos es que cualquier desarrollo se lleva a cabo en relación con una solución pre existente, lo que podría inhibir el surgimiento de una innovación radical. Tampoco es claro hasta cuándo se deben repetir los ciclos de mejora. En el contexto de las asesorías profesionales por proyectos, esto podría traer dificultades para delimitar cuándo termina la asesoría. Del mismo modo, en la enseñanza del diseño no resulta factible evaluar la implementación de las propuestas o medir los efectos de su implementación, de manera que las evaluaciones de los profesores suelen centrarse solo en aspectos formales, estratégicos y persuasivos. Sin embargo, en ámbitos como la educación escolar la lógica de la evaluación y el mejoramiento continuo resultan muy adecuados, 
porque implican aprendizaje. Esto favorece la incorporación de modelos cíclicos en contextos escolares. En efecto, el ciclo de diseño propuesto por el currículum MYP (Middle Years Programme) del International Baccalaureate tiene una estructura circular compuesta de diferentes etapas que se agrupan en cuatro fases principales: investigar, idear o planificar, crear y evaluar (Figura 7a). El modelo Compass de la organización danesa Index Design to Improve Life, también propone una estructura circular de cuatro fases principales - preparar, percibir, prototipo y producir-, cada una compuesta por acciones o actividades (Figura 7b). El sistema incorpora las tres dimensiones de sostenibilidad (social, ambiental y económica) y establece los parámetros de evaluación (forma, impacto y contexto). Además, define los objetivos de aprendizaje de cada fase, describe las actividades y propone técnicas para facilitar su implementación. Es una propuesta muy interesante, que logra articular elementos desde el diseño, la educación y la sostenibilidad (Bravo, 2016).

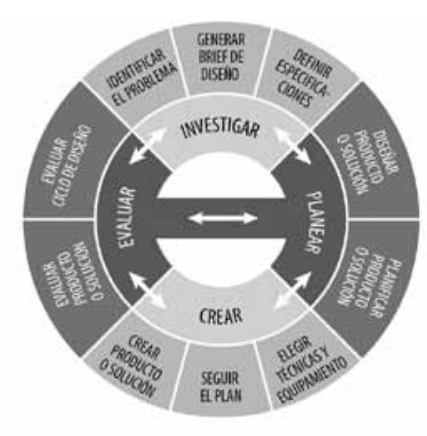

a) Ciclo de diseño según MYP, International Baccalaureate

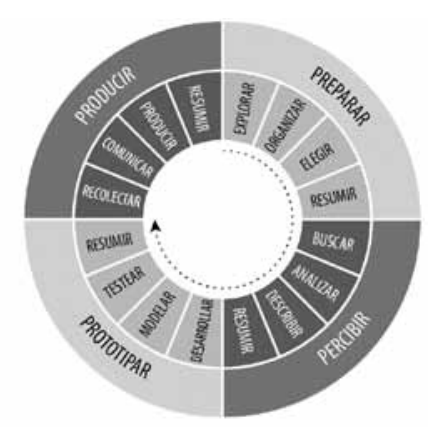

b) Modelo Compass de Index Design to Improve Life

Figura 7: Procesos de diseño con estructura cíclica adaptados al contexto educativo.

\section{Conclusión}

En los últimos años el design thinking ha trascendido las fronteras de la disciplina del diseño, siendo difundido entre profesionales de otras áreas como un método de resolución creativa de problemas. En este contexto, ha sido frecuente el uso de representaciones del proceso de diseño, porque explican de manera didáctica sus etapas, ciclos de iteración y tipos de pensamiento involucrados. Sin embargo, estos modelos no son reflejos neutros de la realidad, ya que la acción de representar supone seleccionar y enfatizar algunos aspectos del fenómeno representado, pero también omitir y neutralizar otros. En esta operación los recursos visuales contribuyen a expresar distintos atributos y narrativas, mediante formas, estructuras, direcciones, colores, etc. De esta manera la representación opera clasificando, 
conceptualizando y asignando valores que son producidos, socializados y reproducidos; así contribuye a la configuración de una determinada "cultura del diseño" y genera acciones que intervienen la realidad, a través de la ejecución de procesos de diseño.

En este artículo nos preguntamos acerca de las concepciones o narrativas subyacen a esos modelos, cuestión que quisimos abordar desde la perspectiva de las metáforas, entendidas como una comparación tácita que impregna al diseño de significados provenientes de otros fenómenos de la realidad. La relevancia de esta pregunta radica en el uso didáctico que se da a estos modelos, en la medida que no solo tienen la función de representar cómo es el proceso de diseño, sino también de guiar su ejecución. Como resultado identificamos cuatro categorías metafóricas que agrupan diferentes representaciones visuales del proceso de diseño: a) el diseño como un proceso racional; b) el proceso de diseño como la trayectoria del pensamiento; c) el diseño como proceso de evolución y d) el proceso de diseño como ciclo de aprendizaje y mejora. Cada una de ellas enfatiza algún aspecto del diseño y en consecuencia utiliza recursos gráficos que contribuyen a comunicar dichos elementos.

La selección de modelos del proceso de diseño para guiar la ejecución de un proyecto, tanto en el contexto profesional como educativo, en cualquiera de sus niveles -desde la escuela hasta la universidad, desde el pregrado hasta la educación continua, desde el postgrado hasta la capacitación de la fuerza laboral- requiere considerar las características del modelo, sus atributos, narrativas y significados implícitos, porque no es lo mismo el diseño entendido como un diagrama de flujo o como un ciclo de mejora continua.

Convertirse en profesional implica desarrollar y refinar la práctica profesional que integra conocer, actuar y estar en el mundo (Dall'Alba, 2009). Argumentamos que los modelos o representaciones visuales del proceso de diseño actúan como dispositivos didácticos al mediar entre los objetivos de la instrucción y sus resultados (Gellert, 2004). Asimismo, estos modelos contribuyen a producir y reproducir una cultura profesional del diseño, en la medida que su creación, difusión y consumo, permiten transmitir valores compartidos y una narrativa entre los diseñadores y otras personas que desean adoptar esta forma de trabajar, pensar o aprender.

\section{Notas}

1. También es importante considerar que la representación visual de la temporalidad está estrechamente relacionada a la dirección de la escritura y de la lectura, de manera que no puede ser asumida como universal (Kress \& van Leeuwen, 2006). Tversky (2001) sugirió que aquellos que escriben y leen de derecha a izquierda también tienden a mapear conceptos temporales de derecha a izquierda. En esta misma línea, Mijksenaar (1974) relata la experiencia de mineros africanos que leyeron de derecha a izquierda instrucciones visuales diseñadas para ser leídas de izquierda a derecha, dado lo cual invirtieron el orden de las acciones que debían realizar, y en lugar de cargar piedras, las descargaron.

2. De acuerdo con la categorización de imágenes didácticas propuesta por Díaz-Barriga y Hernández (2002), la mayor parte de las representaciones del proceso de diseño son de 
tipo algorítmica, porque definen pasos para resolver un problema. Los diagramas más complejos representan diferentes formas de pensamiento -como el diagrama del doble diamante- o las dimensiones involucradas en el proceso diseño -como el modelo de Kuman-, podrían considerarse también de tipo funcional.

\section{Referencias bibliográficas}

Akin, Ö. (1986). A formalism for problem restructuring and resolution in design. Environment and Planning B: Planning and Design, 13(2), 223-232. https://doi.org/10.1068/ b130223

Alexander, C. (1973). Notes on the Synthesis of the Form (7th ed.). Cambridge, Massachusetts: Harvard University Press.

Banks, M. (2007). Using visual data in qualitative research. London: SAGE Publications.

Bason, C., \& Austin, R. D. (2019). The Right Way To Lead Design Thinking. Harvard Business Review, 97(2), 82-91.

Beausoleil, A. M. (2016). The case for design-mediated innovation pedagogy. The University of British Columbia.

Bobbe, T.; Krzywinski, J. \& Woelfel, C. (2016). A comparison of design process models from academic theory and professional practice. Proceedings of International Design Conference, DESIGN, DS 84, 1205-1214.

Bonsiepe, G. (1999). Del objeto a la interfase: mutaciones del diseño. Buenos Aires: Infinito.

Bowker, G. C. \& Star, S. L. (2000). Sorting Things Out: Classification and Its Consequences. Cambridge, Massachusetts: MIT Press.

Bravo, U. (2016). Visual analogies: representation of the design process and its application in the field of education. Base Diseño e Innovación, 2, 42-49.

Costa, J. (1998). La esquemática. Visualizar la información. Barcelona: Paidós.

Council Design, Kolarz, P.; Simmonds, P. O.; Francis, C.; Kovacs, H.; Sharp, T. \& Wain, M. (2015). Innovation by Design. Retrieved from https://www.designcouncil.org.uk/sites/ default/files/asset/document/innovation-by-design.pdf

Cross, N. (2005). Engineering Design Methods Strategies for Product Design (4th ed.). Chichester, England: John Wiley \& Sons.

Cross, N. (2013). Design thinking: Understanding how designers think and work (2nd ed.). London and New York: Bloomsbury Academic.

Dall'Alba, G. (2009). Learning professional ways of being: Ambiguities of becoming. Educational Philosophy and Theory, 41(1), 34-45. https://doi.org/10.1111/j.14695812.2008.00475.x

Díaz-Barriga, F. \& Hernández, G. (2002). Estrategias docentes para un aprendizaje significativo. Una interpretación constructivista (2nd ed.). México: McGraw Hill.

Dorst, K. (2011). The core of "design thinking" and its application. Design Studies, 32(6), 521-532. https://doi.org/10.1016/j.destud.2011.07.006

Dorst, K. \& Cross, N. (2001). Creativity in the design process: co-evolution of problemsolution. Design Studies, 22, 425-437. https://doi.org/10.1016/S0142-694X(01)00009-6 
Dubberly, H. (2005). How do you design? A compendium models. https://doi.org/10.1016/j. bbamcr.2006.09.022

Dubberly, H.; Evenson, S. \& Robinson, R. (2008). The Analysis-Synthesis Bridge Model. Interactions, March-Ap, 1-5. https://doi.org/10.1145/1340961.1340976

Gellert, U. (2004). Didactic material confronted with the concept of mathematical literacy. Educational Studies in Mathematics, 55(1-3), 163-179. https://doi.org/10.1023/ B:EDUC.0000017693.32454.01

Gericke, K. \& Blessing, L. (2012). An analysis of design process models across disciplines. Proceedings of International Design Conference, DESIGN, DS 70, 171-180.

Hall, S. (1997). Representation: Cultural representations and signifying practices (Vol. 2). London, California, New Delhi: Sage.

Hassi, L. \& Laakso, M. (2011). Conceptions of Design Thinking in the Design and Management Discourse. Proceedings of IASDR2011, the 4th World Conference on Design Research, 1-10.

Holliday, A. (1999). Holliday 1999 p. 237 small culture.pdf. Applied Linguistics, 20(2), 237-264.

Howard, T. J.; Culley, S. J. \& Dekoninck, E. (2008). Describing the creative design process by the integration of engineering design and cognitive psychology literature. Design Studies, 29(2), 160-180. https://doi.org/10.1016/j.destud.2008.01.001

Jermier, J. M. \& Forbes, L. C. (2011). Metaphor as the foundation of organizational studies: Images of organization and beyond. Organization and Environment, 24(4), 444-458. https://doi.org/10.1177/1086026611436328

Johansson-Sköldberg, U.; Woodilla, J. \& Cetinkaya, M. (2013). Design Thinking: Past , Present and Possible Futures. Creativity and Innovation Management, 22(2), 121-146. https://doi.org/10.1111/caim.12023

Koberg, D. \& Bagnall, J. (1981). The all new universal traveler: A soft-systems guide to creativity, problem-solving, and the process of reaching goals. Altos, California: William Kaufmann Inc.

Kress, G., \& van Leeuwen, T. (2006). Reading images. The grammar of visual design (2nd ed.). London: Taylor \& Francis e-Library.

Kumar, V. (2012). 101 design methods: A structured approach for driving innovation in your organization. John Wiley \& Sons.

Lawson, B. (2004). What Designers Know. Oxford: Architectural Press.

Lawson, B. (2005). How Designers Think. The design process demystified (4th ed.). Oxford: Architectural Press.

Maher, M. L.; Poon, J. \& Boulanger, S. (1996). Formalising design exploration as co-evolution: A combined gene approach. Advances in Formal Design Methods for Computer-Aided Design, 3-30. https://doi.org/10.1007/978-0-387-34925-1

McComb, C.; Kotovsky, K. \& Cagan, J. (2014). Quantitative comparison of high-and lowperforming teams in a design task subject to drastic changes. Proceedings of the ASME 2014 International Design Engineering Technical Conferences and Computers and Information in Engineering Conference. Buffalo, NY: ASME 2014.

Meirelles, I. (2013). Design for information: an introduction to the histories, theories, and best practices behind effective information visualizations. Beverly, MA: Rockport publishers.

Mijksenaar, U. (1974). Signposting and Communication Media. Icographic, 7, 15-21. 
Morgan, G. (1997). Images of Organization. Thousand Oaks, California: Sage.

Rittel, H. \& Webber, M. (1973). Dilemmas in a General Theory of Planning. Policy Sciences, 4(2), 155-169. Retrieved from https://dbproxy.udallas.edu/login?url=http://search.ebsco host.com/login.aspx?direct $=$ true $\& d b=b$ th $\& A N=16620094 \&$ site $=$ ehost-live\&scope $=$ site

Teal, R. (2010). Developing a (Non-linear) Practice of Design Thinking. International Journal of Art and Design Education, 29(3), 294-302. https://doi.org/10.1111/j.14768070.2010.01663.x

Tversky, B. (2001). Structures of mental spaces. Proceedings 3rd International Space Syntax Symposium Atlanta, 11-12. Citeseer.

Ulrich, K. T. \& Eppinger, S. D. (2000). Product Design and Development. Singapore: McGrawHill Higher Education.

Wilkes, P. (1989). Images of Organization: An Essay Review. Journal of Educational Administration, 27(2), 67-71.

Wölfel, C.; Debitz, U.; Krzywinski, J. \& Stelzer, R. (2012). Methods use in early stages of engineering and industrial design - A comparative field exploration. Proceedings of International Design Conference, DESIGN, DS 70, 1397-1404.

Wynn, D. C. \& Clarkson, P. J. (2005). Models of Design. In J. Clarkson \& C. Eckert (Eds.), Design process improvement: a review of current practice (pp. 34-59). London: Springer Science \& Business Media.

\begin{abstract}
The visual representations of the design process are used as didactic devices to illustrate) which are the main stages, the types of thought involved, activities and the feedback loops that take place throughout the process. Considering that the action of representing consists of selecting or emphasizing some elements of the phenomenon represented by others, we ask ourselves which conceptions of design communicate those models and which aspects they omit. We propose a theoretical study to discuss two central elements: a) the didactic function of these models and b) the different narratives using metaphors that these models express. We have selected, at our discretion, different representations of the design process from academic and professional sources, including articles and books in physical and digital formats. We conclude that these models contribute to produce and reproduce a professional culture of design, to the extent that its creation, dissemination, and consumption, facilitating transmitting "shared values" and a "narrative" between designers and others who seek to adopt the way of working, think or learn from the designers.
\end{abstract}

Keywords: design thinking - design process - visual representation - didactic image.

Resumo: Representações visuais do processo de design são usadas como dispositivos didáticos para ilustrar quais são as principais etapas, os tipos de pensamento envolvidos e os ciclos de feedback que ocorrem ao longo do processo. Considerando que o ato de representar implica selecionar ou enfatizar alguns elementos do fenômeno representado 
acima de outros, nos perguntamos quais conceitos de design comunicam esses modelos e quais aspectos eles omitem. Propomos um estudo teórico com o objetivo de discutir dois aspectos centrais: a) a função didática desses modelos eb) as diferentes narrativas ou metáforas que eles expressam. Selecionamos diferentes representações do processo de design de fontes acadêmicas e profissionais, incluindo artigos e livros em formato físico e digital. Concluímos que esses modelos contribuem para a produção e reprodução de uma cultura profissional de design, na medida em que sua criação, disseminação e consumo permitem a transmissão de "valores compartilhados" e uma "narrativa" sobre design entre designers e outras pessoas que buscam adotar o design, maneira de trabalhar, pensar ou aprender com os designers.

Palavras chave: design thinking - processo de design - representação visual - imagem didática.

[Las traducciones de los abstracts fueron supervisadas por el autor de cada artículo] 